minimal. Carefully constructed clinical trials are required to compare the effects of present treatment strategies with more conservative approaches. Clinical and echocardiographic PDA scoring systems should be incorporated into the routine care of preterm infants and used to justify any treatment undertaken.

\section{G247(P) ANTENATAL DETECTION AND 30-DAY MORTALITY IN CRITICAL CONGENITAL HEART DISEASE WITH TWO VENTRICLE OUTCOME A POPULATION-BASED STUDY}

${ }^{1} \mathrm{~K}$ Khor, ${ }^{1} \mathrm{AK}$ Finucane, ${ }^{2} \mathrm{~L}$ Sadler, ${ }^{2,3} \mathrm{E}$ Parry, ${ }^{1} \mathrm{~J}$ Wright, ${ }^{4} \mathrm{~J}$ Beca, ${ }^{5} \mathrm{E}$ Cloete, ${ }^{1} \mathrm{TL}$ Gentles. ${ }^{1}$ Paediatric Cardiac and Congenital Service, Starship Hospital, Auckland, New Zealand; ${ }^{2}$ National Women's Hospital, Auckland City Hospital, Auckland, New Zealand; ${ }^{3}$ Greenlane Paediatric and Congenital Cardiac Service, Starship Hospital, Auckland, New Zealand; ${ }^{4}$ Paediatric Intensive Care, Starship Hospital, Auckland, New Zealand; ${ }^{5}$ Liggins Institute, University of Auckland, Auckland, New Zealand

\subsection{6/archdischild-2018-rcpch.240}

Introduction Isolated reports from selected populations suggest early diagnosis in readily treatable critical congenital heart disease (cCHD) result in improved outcome, especially where the surgical outcome is excellent.

However, population-based data are sparse and its interpretation complicated by inclusion of complex heart disease and noncardiac comorbidity that may independently influence outcome.

Aim Compare the outcome of infants born with critical CHD (CHD resulting in operation or death by 30 days) and likely resultant two-ventricle circulation from those born 2006-2010 with 2011-2014, in relation to antenatal diagnosis and 30 day mortality.

Methods A population-based retrospective review of critical CHD, with case ascertainment via the National Fetal Cardiology, the Cardiac Surgical and Governmental mortality review databases.

Results Of the 436 infants born with critical CHD and a potential 2 ventricle circulation, 371 did not have a major non-cardiac abnormality or syndrome.

The proportion of critical CHD's diagnosed antenatally increased during the study period $(\mathrm{p}=0.006)$.

The 30 day mortality declined from $7.0 \%$ to $0.9 \%(p=0.049)$. Mortality was largely confined to those who died prior to surgery.

Conclusions There has been a significant increase in the rate of antenatal diagnosis in infants with readily treatable critical CHD.

This has been an associated a decrease in the 30 day mortality.

Earlier recognition of cCHD resulting in appropriate delivery location and a reduced risk of circulatory collapse has very likely contributed to the improved survival.

\section{G248(P) SIGNIFICANT FINDINGS? DIFFICULTIES TRIAGING REFERRALS OF CHILDREN WITH HEART MURMURS}

J Round, E Cartwright. Department of Paediatrics, St George's Hospital NHS Trust, London, UK

10.1136/archdischild-2018-rcpch.241
Aims Typical primary care practice is to refer all children with persistent heart murmurs for further assessment. Although most of these murmurs are benign, those with serious underlying pathology are at risk of having their care delayed because of the number of patients referred. This practise may also lead to unnecessary anxiety for parents and patients.

We sought to analyse GP referral letters to establish if patients at risk of structural disease could be identified to enable them to be seen sooner.

Method GP referral letters to a tertiary hospital paediatric cardiology clinic requesting evaluation of a murmur of a tertiary hospital in the period from January 2016 to May 2017 $(n=90)$ were identified. They were analysed for features suggestive of significance of the murmur. These were then compared to the echocardiography findings in the subsequent clinic letter.

Results Of the letters analysed, $10 \%$ of patients had underlying structural abnormalities. 5/90 referrals (5.5\%) specified a grade of murmur, and $31 / 90$ (34\%) specified a location of the murmur. $23 / 90(25.5 \%)$ referrals mentioned the presence or absence of associated features.

No characteristics of the murmur were associated with the presence of a structural cause.

Conclusion This study demonstrates that it is currently impossible to prioritise patients based on information provided in GP referral letters. This suggests either provision of cardiology screening outpatient clinics should be increased, or more attention paid to learning about children's heart disease in undergraduate and GP curricula. Referral using a specific form may also prompt referrers to seek specific findings and improve triage.

\section{G249(P) ATRIAL ARRHYTHMIAS FOLLOWING UMBILICAL CATHERISATION}

B Saha, A Mishra, S Konnuru. Neonatology, Royal Bolton Hospital, Manchester, UK

\subsection{6/archdischild-2018-rcpch.242}

Case description An infant born at 35 weeks gestation to an insulin dependent diabetic and hypertensive mother was admitted to neonatal unit due to respiratory distress and hypoglycaemia. UVC was inserted as blood sugars were persistently below $2 \mathrm{mmol} / \mathrm{L}$. Soon after the UVC placement baby became increasingly tachycardic with a heart rate greater than 200 per minute with narrow complex tachycardia on the ECG. The UVC was pulled back from its initial high position within the cardiac silhouette and subsequently removed but the tachycardia persisted. There was no response to IV Adenosine and Amiodarone, and a second ECG revealed Atrial Flutter. Serum biochemistry was normal and an Echocardiogram did not reveal anatomical abnormalities. Synchronised shock of 0.5 Joules $/ \mathrm{kg}$ restored sinus rhythm and the baby remained in sinus rhythm till discharge without requiring any further treatment.

Conclusion There are only few reports in literature of atrial flutter following UVC insertion. Mother was diabetic which was an additional risk factor for neonatal arrhythmias.Electrical cardioversion has good effect in restoring sinus rhythm and maintenance antiarrhythmics are usually not needed as risk of recurrence is low. 